\title{
Willkommen im Club! Eine Anregung zu mehr Kontingenzfreudigkeit in der qualitativen Sozialforschung - Kommentar zu A. Nassehi und I. Saake in ZfS 1/2002 \\ Welcome to the Club! A Plea for Even More Contingency in Qualitative Research (Comment on Nassehi and Saake in ZfS 1/2002)
}

\author{
Stefan Hirschauer \\ Institut für Soziologie, Ludwig-Maximilians-Universität München, Konradstraße 6, D-80801 München
}

\section{Jörg Bergmann}

Fakultät für Soziologie, Universität Bielefeld, Postfach 1001 31, D-33501 Bielefeld

Anders als narrative Interviews gehören die Aufsätze einer Fachzeitschrift nicht zu einem Typ von „Kommunikation, die vom Risiko der Ablehnung befreit ist". Armin Nassehi und Irmhild Saake (AN/ IS) haben dies bereits in der Begutachtung ihres Manuskripts für die ZfS ertragen und genutzt, freilich nicht so weit, dass wir auf einen Schritt aus dem anonymen ,peer review' in einen ,open peer commentary“ (S. Harnad) verzichten wollen.

Unser Argument sei in Kürze vorweggenommen: AN/IS überziehen ihren Claim, indem sie ein für die Biographieforschung u.E. überzeugendes Argument auf die Ethnomethodologie und die qualitative Sozialforschung insgesamt ausdehnen. Dabei unterlaufen ihnen nicht nur gravierende Rezeptionsfehler, sie übersehen auch, dass andere Ansätze in der qualitativen Sozialforschung seit langem in eben ihrem Sinne argumentieren und forschen. Verglichen mit ihnen zeigt sich das empirische Vorgehen von AN/IS erstaunlich kontingenzscheu.

Rekonstruieren wir kurz den Aufsatz von AN/IS. Er hat den großen Vorzug, methodische Fragen vom Verhältnis theoretischer und empirischer Soziologie anzugehen und konfrontiert dabei das epistemologische Selbstverständnis vieler empirischer Sozialforscher mit höheren Reflexivitätsanforderungen. Zugleich wird ein Brückenschlag geboten: Versprochen wird, dass ein beobachtungstheoretischer Ansatz, der bislang nicht weiter durch Empirieoffenheit aufgefallen war, doch irgendetwas mit der Praxis empirischer Forschung zu tun haben will.

Leider wird dies den LeserInnen kaum stichhaltig an empirischem Material demonstriert (hier wird man eher auf Geduld eingestimmt), AN/IS begnügen sich primär mit dem einfacheren Unterfangen, den status quo der qualitativen Sozialforschung zu kritisieren. Dabei scheinen uns ihre Argumente gültig und originell für die Biographieforschung und deren doppelt subjektzentriertes Verfahren des (narrativen) Interviews. AN/IS entwickeln eine verstehensskeptische Position, die einen kommunikationsanalytischen Blick für eine konsequente Symmetrisierung von Interviewer und Interviewtem nutzt. Sie reflektieren darauf, was für kommunikative Artefakte durch eine soziologisch veranstaltete Gesprächssituation geschaffen werden: Nicht das narrative Interview sei seinem Gegenstand ,naturalistisch' angemessen, sondern die Narration sei das Artefakt einer vom Dissens befreiten Kommunikationssituation. Diese bringt alle möglichen kommunikativen Ereignisse hervor, für deren Untersuchung den AutorInnen offenbar eine Art Diskursanalyse vorschwebt, die die Äußerungen von Informanten aus einem subjekttheoretischen ,Deutungskäfig' befreit und vor autoritativen Deutungsakten bewahrt, mit denen sie auf einen (Tief-)Sinn verpflichtet werden, den ihr Urheber nicht kontrollieren kann. Weder muss man diese Äußerungen mit dem Gewicht der Individualgeschichte eines Menschen belasten, noch sollte ihre Analyse das Sprachspiel des Sprechers auf Narration beschränken. Stattdessen kann man bescheidener - an der Textoberfläche verharren und alle „Formen der Sagbarkeit" betrachten, in denen ein Thema zur Sprache gebracht wird - mit einem „funktionale(n) Verfahren, das auf strikte Kausalannahmen ebenso verzichtet wie auf die innere Unendlichkeit subjektiven Sinn generierender Handelnder" (83).

Wir halten diese Argumente für trifftig. (Und es wäre auch nicht unsere Aufgabe, sie von ihrer überschießenden Polemik zu befreien). Unser Einspruch 
gilt vielmehr AN/IS Versuch, ihre Argumentation gegen den Naturalismus und Humanismus der Biographieforschung pauschal auf die gesamte qualitative Sozialforschung auszudehnen. AN/IS legen dar, dass die Schulenbildung in der qualitativen Sozialforschung (ebenso wie die Dichotomie von ,Quantitativem“ und,Qualitativem') von Argumentationszwängen entlastet, indem sie einen Projektionsschirm für Probleme der je eigenen Seite aufbaut. Die Dichotomisierung von Ansätzen entlastet in der Tat, aber ihr oberflächliches Zusammenwerfen tut dies auch. Die Frage ist halt, ob die Abstraktionsgewinne die Differenzierungsverluste aufwiegen. Eben dies möchten wir bestreiten.

Zunächst zu den Möglichkeiten des Abstraktionsgewinns: Die Heterogenität der qualitativen Sozialforschung wird von AN/IS gleich eingangs trivialisiert, sie suchen einen abstrakten Gesichtspunkt, von dem aus nicht nur deren unübersichtliche Mannigfaltigkeit, sondern auch die Differenz zur quantitativen Sozialforschung und schließlich auch die zur theoretischen Soziologie zweitrangig werden. AN/IS sehen diesen Gesichtspunkt einer aller Soziologie gemeinsamen „Logik der Forschung“ im Umgang mit Kontingenz. Lassen wir uns also einmal darauf ein, dass die ,Kontingenzbeobachtung' eine interessante Formel für das Selbstverständnis soziologischer Forschung sein könnte. Die Feststellung, dass alle Sinnproduktion prinzipiell unabgeschlossen ist und weder notwendige noch beliebige Resultate hat, ist freilich so allgemein, dass auf Anhieb kaum zu sehen ist, ob aus ihr spezifische Konsequenzen für die Forschungspraxis folgen können, oder ob sie ähnlich unverbindlich bleiben muss wie Einsichten z. B. in die Unterscheidungsabhängigkeit der Weltkonstitution oder die Theoriegeladenheit aller Beobachtung. Diese Frage drängt sich vor allem dann auf, wenn der Kontingenzbegriff wie bei AN/IS durch das Suffix „Vernichtung“ bzw. „Beobachtung “ mit einer klaren Wertpräferenz ausgestattet wird. Das klingt zwar schön kämpferisch, damit es aber überhaupt für empirische Forschung Sinn machen kann, scheinen uns mindestens zwei Differenzierungen nötig: Zum einen die zwischen der (kritisierten) Kontingenzvernichtung durch die soziologischen Beobachter und der (gar nicht thematisierten) durch die Teilnehmer. Alle Kommunikation ist darauf angewiesen, zumindest punktuell oder für bestimmte Zwecke immer wieder einmal Sinnfestlegungen vorzunehmen. Soziologische Forschung soll dies nun nicht einfach (im Anschluss an das Alltagswissen) mitvollziehen, sondern in der Tat beobachten. Das heißt aber, sie beobachtet immer sowohl Kontingenz als auch „Kontingenzvernichtung “ (etwa in einem Gesprächsverlauf).
Zum anderen braucht es eine Differenzierung zwischen einer kontingenzbeobachtenden Frühphase der Forschung und einer kontingenzreduzierenden Phase disziplinärer Kommunikation. Auch die ,Beobachter' sind ja, sobald sie soziologisch über Kommunikation reden wollen, Teilnehmer kommunikativer Prozesse, die zu gewissen Sinnfestlegungen nötigen (solange man einen minimalen Anspruch hegt, ,verstanden' zu werden). Auch die rezeptive Kontingenzbeobachtung mündet also in Formen der Kontingenzbewältigung, nämlich in empirisch gesättigte Argumentationsmuster disziplinärer Kommunikation, z. B. jenen wuchtigen Idealtypen, die AN/IS am Ende ihres Aufsatzes vorstellen, ohne darin irgendein Problem zu sehen.

AN/IS zeigen sich an solchen weiterführenden Fragen desinteressiert, da sie den Kontingenzbegriff wie gesagt vor allem in einer polemischen Funktion gebrauchen. Und damit ist auch schon von den Differenzierungsverlusten zu reden:

1. Die empirische Sozialforschung geht sicher nicht gleichermaßen in Kommunikation auf wie theoretische Soziologie, aber wir sehen die Sozialwissenschaften von vielen anderen Wissenschaften dadurch unterschieden, dass schon in ihrem Gegenstandsbereich Unterscheidungen kommunikativ gehandhabt werden. Der Gegensatz ihrer methodischen Paradigmen entzündet sich nun aber eben daran, wie mit diesen ,Konstruktionen erster Ordnung' umgegangen wird: indifferent-definitorisch oder rezeptiv-rekonstruktiv. AN/IS meinen, diese Differenzierung übergehen zu können, weil sie schon die zwischen theoretischer und empirischer Soziologie leichtfüßig mit der Feststellung überspringen, dass beide insofern eine „Methode der Beobachtung “ darstellen, als sie „Unterscheidungen handhaben " müssen. Das ist in der Tat so, aber kann man übergehen, wie verschieden es geschieht? Die Formel von den „Begriffe(n) als Entscheidungen " trifft am besten für den von AN/IS verwendeten Theorieansatz zu: Die Kontingenz der Begriffssetzung ist in der Systemtheorie Luhmanns in der Tat gewaltig, da sowohl die Artifizialität als auch die Selbstbezüglichkeit der Sprache immens gesteigert wurden, so dass schon die zahllosen internen Anschlusspflichten ausreichen, Beliebigkeit (die andere Seite der Kontingenzvernichtung) zumindest ein wenig einzuschränken. (Dies sei hier übrigens ganz unpolemisch gesagt: Die Soziologie soll sich durchaus einen Theorietypus leisten, dessen Aussagen als - mehr oder weniger gelungene - Erfindungen zu bewerten sind.) Auch die quantitative Sozialforschung hat eine Affinität zu diesem Modus des Unterscheidungsgebrauchs, insofern die Belie- 
bigkeit ihrer gesetzten Definitionen durch Selbstreferentialität (nämlich deren Verwendung in früheren und parallelen Forschungen) eingeschränkt wird. Freilich machen sich hier bereits pragmatische Zwänge empirischen Forschens bemerkbar, wenn Operationalisierungen nach Bindungen an die Alltagssprache verlangen (damit die Befragten den Fragebogen überhaupt verstehen). In der qualitativen Sozialforschung setzt man Begriffe dagegen wie die eigenen Füße in ein Feld, von dem man annimmt, dass es bereits von den Fußspuren der ,Eingeborenen' übersät ist, für die man sich interessiert. (Eben dafür steht etwa ein Begriff wie Blumers ,sensitizing concepts'). Deren ,Exotisierung' ist nicht bloß (wie AN/IS suggerieren) ein Interviewartefakt, es ist vielmehr eine Heuristik des Unbekannten (Amann/Hirschauer 1997; 1999), mit der sich die Soziologie überhaupt empirisch gegenüber dem offen halten kann, was sie theoretisch immer schon ,besser weiß‘. Nur durch Aufrechterhaltung und Steigerung solcher Differenzen kann Empirie ein maximales Irritationspotential in soziologischen Wissensprozessen entfalten.

2. AN/IS sind nicht nur diesen Differenzierungen gegenüber indifferent, sie differenzieren auch nicht zwischen unterschiedlichen Typen von Kommunikation. Methodische Kontrolle könne „nur noch heißen: Einsicht in die epistemologische Verschlingung von Forschung und Gegenstand ...“ (81), die sich um ,Gegenstandsadäquatheit" nicht mehr zu scheren brauche, da eben alle Methoden ihre Gegenstände konstituieren. In der Nacht der Kontingenz sind alle Daten grau: anspruchslos empiristisch und ein bisschen langweilig. Aber soll aus dieser ,Einsicht' nun folgen, dass man Konversationen genausogut mit Interviews, nonverbale Kommunikation mit Audioaufzeichnungen, politische Meinungen mit teilnehmender Beobachtung erheben kann? - wenn man nur anschließend schlau beobachtet, was „die Daten beobachten“? Sicher kann man seine Zeit damit verbringen, fragwürdig erhobene Daten interpretativ zu ,retten' (und man muss dazu nicht einmal den Lehnsessel verlassen!), aber wenn man innerhalb sozialwissenschaftlicher Kommunikation (über Kommunikation) Anschlussbereitschaft erzielen will, so wird man auch aus dem Passungsverhältnis von Methoden und Gegenständen noch ein paar handwerkliche Argumente entwickeln müssen, um gute von schlechten Daten unterscheiden zu können.

3. Nun lässt sich AN/IS plakative Gegenüberstellung von „Kontingenzbeobachtung und -vernichtung " freilich ihrerseits als Qualitätskriterium für unterschiedliche Datentypen fruchtbar machen.
Nennen wir es die ,differenzielle Kontingenzfreudigkeit $^{\star}$ qualitativer Forschungsstrategien. Dann muss man die Abstraktion von deren Pluralität aber zurücknehmen. AN/IS plausibilisieren ihren Vorwurf der „Kontingenzvernichtung “ ja an einem besonderen Fall von qualitativer Sozialforschung: der mit narrativen Interviews arbeitenden Biographieforschung.

Diese Verengung ist in zweierlei Hinsicht problematisch. Zum einen, weil AN/IS meinen, durch die abstrakte Fassung ihres Einwands alle Ansätze qualitativer Sozialforschung mit der Biographieforschung identifizieren zu können. Der gesamte Aufsatz ist von einer Gleichsetzung qualitativer Sozialforschung mit (narrativen) Interviews durchzogen. AN/IS können sich offenkundig gar nicht vorstellen, dass man auch auf andere Weise Daten generiert (mittels Aufzeichnungen, Beobachtungen, Videokameras, Dokumentenerhebungen, Gruppendiskussionen usw.). Man kann aber nicht „die“ qualitative Sozialforschung auf die sozialen Eigenschaften der Interviewsituation zurückführen, wenn sie gar nicht (und z. T. erklärtermaßen nicht) mit Interviews arbeitet. ${ }^{1}$

Zum anderen wirft die Verengung auf Biographieforschung ein immanentes Problem auf. Die Argumentation von AN/IS hat ihren größten blinden Fleck darin, dass die AutorInnen ihr Plädoyer für ,Kontingenzerhaltung' partout für ein Verfahren der Datengenerierung halten, das ,unrettbar' durch Kontingenzvernichtung charakterisiert ist: eben das Interview. Interviews steuern Sinnbildungsprozesse massiv durch Fragestimuli, Interaktionsformate und die Situationsrahmung. Eben dies demonstrieren AN/IS ein weiteres Mal, ohne jedoch die Konsequenz zu ziehen, sich Verfahren zuzuwenden, die kontingenzfreudiger Daten generieren als es die Frage-Antwort-Zwänge eines Zwiegesprächs tun. Wenn man etwa in der Konversationsanalyse rekonstruktive Daten strikt ablehnt, macht man sich unterschiedslos offen für alles im Rahmen der Aufzeichnungstechnik beobachtbare Geschehen. „Dass nichts dem Zufall unterliegt“ (75) ist eine methodische Heuristik, die auf eine drastische Beschrän-

\footnotetext{
${ }^{1}$ Im Zusammenhang mit dieser Rezeptionsschwäche sei ein Stilproblem angemerkt: Stimmen AN/IS ihre Leser im Tonfall auf die Vogelperspektive einer grandiosen epistemologischen Supervision ein („eine kommunikations- und beobachtungstheoretische Lektion “ (72), so konfrontieren sie sie der Sache nach mit der Froschperspektive, aus der sie die qualitative Sozialforschung, überblicken'. Insofern müssen wir ein unlösbar scheinendes (gymnastisches) Problem gestehen, mit den Autoren jemals auf gleicher Augenhöhe zu kommunizieren.
} 
kung der hermeneutischen Definitionsmacht zugunsten einer Beobachtung der kontingenten Sinnproduktion durch die Teilnehmer zielt. Geradezu berüchtigt für ihre Kontingenzfreudigkeit ist die Ethnographie: Bis hin zum ,going native ${ }^{\varsigma}$, dem Verlust aller Disziplin, verliert sie sich (in der Phase der Datengenerierung) im Gegenstand, seinen Zufälligkeiten und Gelegenheiten.

AN/IS beschränken sich stattdessen darauf, ihre verstehensskeptische Position (ganz luhmannisch) aus der Begegnung zweier differenter ,Bewusstseine $^{6}$ abzuleiten und zu folgern „es ist die Kommunikation selbst, die die beiden Rollen des Forschers und des Beforschten konstituiert". Warum aber soll man bei dieser Dezentrierung von Personen erst bei der Analyse von Interviewdaten landen, wenn man sie i.S. anderer Ansätze qualitativer Sozialforschung auch schon bei den Strategien der Datengewinnung haben kann? Was nur fixiert eine systemtheoretische (also akteurskritische) Forschungsstrategie auf ein derart akteurzentriertes Verfahren wie das biographische Interview, bei dem sich die Differenz von Alltagswissen und professionellem Wissen ausgerechnet in Personen verkörpert? ${ }^{2}$

Zentraler Effekt dieses letzten Differenzierungsverlustes ist, dass AN/IS übersehen, welche starken Affinitäten ihr eigenes Vorgehen $\mathrm{zu}$ den neben der Biographieforschung ,irgendwie ${ }^{6}$ mitattackierten Formen qualitativer Sozialforschung hat. Diese sind zum Teil durch eben die Argumente motiviert, die die Autoren mit Recht gegen die Biographiefor-

\footnotetext{
${ }^{2}$ Wirklich erstaunlich im Aufsatz von AN/IS ist die Renaissance ganz realistisch unterstellter „Grundstrukturen des Psychischen“ (79). Damit scheint nun etwas ontologisiert zu werden, von dem man nicht geglaubt hätte, dass ausgerechnet soziologische Empirie „bei aller Kontingenz" hier Halt finden soll: Erst werden die Disziplingrenzen mit einer singulären Logik der Sozialforschung hochgehalten, und dann scheint das epistemologische Referenzproblem externalisiert zu werden. Wieso aber sollen die Unterscheidungen des Erzählers jetzt nur mehr dem psychischen System (und seiner ,Sozialisation') zurechenbar sein und nicht etwa Diskursen, die alle möglichen ,Leitunterscheidungen', Mitgliedschaftskategorien und Motivvokabulare (i.S. von C.W.Mills) anbieten? Könnte es sein, dass eine stark an die Bewusstseinsphilosophie anschließende soziologische Theorie (Wagner/Zipprian 1992) zu einer seltsamen Hassliebe gegenüber einer individualistischen Form von Empirie neigt? Solange dürfte ein systemtheoretischer Ansatz freilich auch Schwierigkeiten mit der Subsumption von Datentypen haben, die sich eben nicht umstandslos auf ,Systeme` zurechnen lassen, weil sie von vornherein in Distanz zu solchen Zentrierungen erhoben wurden.
}

schung vorbringen. So liegt eine Konsequenz wie die „dass der Forscher sich von ... der Interviewkommunikation benutzen lassen sollte" (77) ganz auf der Linie des Grundgedankens der Ethnographie seit Malinowski. Die Distanzierung von jedem Bedeutungsessentialismus (80) nachvollzieht für die Biographieforschung, was antihermeneutische und intuitionsasketische Verfahren wie die Konversations- und Diskursanalyse seit langem praktizieren. Dass Texte ihre Kontexte selbst erzeugen (81), liegt der ethnomethodologischen Analyse von institutionellen Gesprächen zugrunde. usw.

Es sind aber nicht nur diese einzelnen Berührungspunkte, es ist vielmehr der ganze Impetus ihrer methodenreflexiven Argumentation, der AN/IS in große Nähe zu dem zweiten Ansatz qualitativer Sozialforschung rückt, von dem sie sich laufend zu distanzieren versuchen: der Ethnomethodologie. Sie selbst formulieren diesen Verdacht gegen sich gleich mehrfach: Es ist die von Aaron Cicourel und Harold Garfinkel gestellte Frage nach der sozialen Logik der Sozialforschung, die sie aufgreifen. Einen Mangel der Ethnomethodologie sehen sie nur darin, dass diese den Blick nicht auch auf sich selbst und ihre Gegenstandskonstitution richte (71). Diesen Mangel erkennen die ,Beobachter' freilich nur, weil sie ihren eigenen Blick offenbar nie gründlich auf die Ethnomethodologie gerichtet haben. ${ }^{3}$ Diese

${ }^{3}$ Im Gegensatz zu den aktuellen Publikationen der Biographieforschung rezipieren AN/IS die Ethnomethodologie über zwei klassische Texte aus den 60er Jahren und drei Handbuchartikel, d.h. kursorisch, hochselektiv und lernblockiert. Das führt zu einer Reihe kurioser Rezeptionsfehler, von denen wir hier nur wenige nennen können: S. 68: Das in den 80er Jahren von Garfinkel postulierte „unique adequacy requirement" hat nichts mit „Regularien“ zu tun, war als Provokation einer gegenstandsvergessenen Methodologie formuliert worden und ist in der Ethnomethodologie mit dem Buch von Lynch (1993) Geschichte. S. 76: Das Konzept des „recipient design“ wird von AN/IS im Kontext einer Diskussion des Interviews eingeführt; von dort kommt es aber weder her noch hat es seinen systematischen Platz dort. S. 77: AN/IS unterstellen der Ethnomethodologie, an der gerade von dieser kritisierten Vorstellung eines „kognitiven Konsenses“ (Thomas Wilson) festzuhalten, und ignorieren damit, mit welcher Radikalität Garfinkel an diesem Punkt über Schütz und über interaktionistische Positionen hinausgegangen ist. S. 78: AN/IS schreiben dem Verfahren der Sequenzanalyse eine kausalanalytische Bedeutung zu, was blanker Unsinn ist. (Umgekehrt ist freilich die Sequenzanalyse prädestiniert, Weisen des Umgangs mit Kontingenz sichtbar zu machen). S. 80: Die Ethnomethodologie nehme Indexikalität nicht ernst genug und halte kommunikative Vagheit für ein Problem sozialer Ordnung? Garfinkel definierte die Ethnomethodologie aber gerade 
hat geradezu ihren Ausgangspunkt in der reflexiven Beobachtung sozialer und sozialwissenschaftlicher Methoden als gleichermaßen Wissen stiftender Praxis. Diese Reflexivität ist ein genuiner Beitrag der Ethnomethodologie zur Soziologie und auch der entscheidende Grund für die z.T. massiven Irritationen, die dieser Ansatz in unserem Fach ausgelöst hat. Die Ethnomethodologie hat sich seit Garfinkel allerdings stark differenziert. Ihr entstammen Forschungslinien, die die Reflexivität des Sozialen fast vollständig als eine Eigenschaft im empirischen Material untersuchen (dies tut etwa die Konversationsanalyse), und solche, die die Selbsteinschließung der soziologischen Beobachtung in den Gegenstand in nachgerade absurde Steigerung getrieben haben (Woolgar 1988, Ashmore 1989, s.a. Pollner 1991). Dazwischen finden sich zahlreiche vermittelnde Positionen (etwa in den studies of work: Lynch 2000).

Inzwischen ist die Ethnomethodologie mit der Radikalität ihres Ansatzes freilich nicht mehr allein, ja sie scheint sogar ganz unverhoffte Anhänger gewonnen zu haben. Zitieren wir dafür einen unverdächtigen Zeugen, der für die Theorie des Autors, auf den AN/IS ihre Argumentation im wesentlichen stützen, einen Titel suchte, der ihn besser kennzeichnet als ,Systemtheorie': „Ich würde vermuten, dass es am ehesten der Titel einer ,reflexiven Soziologie“ ist, der das Unterfangen seiner Soziologie ... trifft. Ich bin mir dabei bewusst, dass ich ihn damit in den heterogenen Zusammenhang einer ,postmodernen' Soziologie rücke; und ich bin mir bewusst, dass seine Soziologie damit Teil eines übergreifenden und nur, ethnomethodologisch' zu nennenden Abenteuers wird, auf das die Soziologie sich einlassen muss, wenn ihr eine relevante Zukunft beschieden sein soll" (Baecker 1999: 36).

Man muss es nicht so emphatisch sagen wie Dirk Baecker, aber zumindest scheint sich der ,Frontverlauf‘ soziologischer Ansätze jenseits von Mikro und Makro, Quantitativ und Qualitativ, System und Akteur bei unbefangenem Blick ebenso aufgelockert wie unübersichtlich darzustellen. Eben dies begrüßen wir auch am Aufsatz von Armin Nassehi und Irmhild Saake.

als Untersuchung der „rational properties of indexical expressions“. Die durch Indexikalität vermittelte Vagheit ist, wie Garfinkel in Übereinstimmung mit Wittgenstein argumentiert und in seinen Demonstrationsexperimenten deutlich macht, eine Bedingung gelingender Kommunikation. usw. usw.

\section{Literatur}

Amann, K. / Hirschauer, S., 1997: Die Befremdung der eigenen Kultur. Ein Programm. S. 7-52 in dies. (Hg.) Die Befremdung der eigenen Kultur. Frankfurt: Suhrkamp.

Amann, K. / Hirschauer, S., 1999: Soziologie treiben. Für eine Kultur der Forschung. Soziale Welt 50: 495-506.

Ashmore, M., 1989: The Reflexive Thesis: Wrighting Sociology of Scientific Knowledge. University of Chicago Press.

Baecker, D., 1999: Wenn etwas der Fall ist, steckt auch etwas dahinter. S. 35-48 in R. Stichweh (Hrsg.) Niklas Luhmann - Wirkungen eines Theoretikers. Bielefeld: transcript Verlag.

Lynch, M., 1993: Scientific Practice and Ordinary Action. Ethnomethodology and Social Studies of Science. New York: Cambridge University Press.

Lynch, M., 2000: Against Reflexivity as an Academic Virtue and Source of Privileged Knowledge. Theory, Culture \& Society 17: 26-54.

Pollner, M., 1991: ,Left‘ of Ethnomethodology. The Rise and Decline of Radical Reflexivity. American Sociological Review 56: 370-380.

Wagner, G. / Zipprian, H., 1992: Identität oder Differenz? Bemerkungen zu einer Aporie in Niklas Luhmanns Theorie selbstreferentieller Systeme. Zeitschrift für Soziologie 21: 394-405.

Woolgar, S., 1988. Knowledge and Reflexivity. New Frontiers in the Sociology of Knowledge. London: Sage. 